\title{
Niveles de perfil lipídico en pacientes con VIH-sida en tratamiento con efavirenz y atazanavir. Estudio de cohorte
}

Fernando Geldres-Molina, ${ }^{1}$ Alex Castañeda-Sabogal, ${ }^{1}$ Maryori M. Hilario-Gómez ${ }^{2 *}$ y Joshuan J. Barboza ${ }^{3}$ ${ }^{1}$ Universidad Privada Antenor Orrego, Facultad de Medicina, Trujillo; ${ }^{2}$ Universidad Nacional Mayor de San Marcos, Sociedad Científica de San Fernando, Lima; ${ }^{3}$ Universidad Señor de Sipán, Chiclayo. Perú

\section{Resumen}

Introducción: El tratamiento antirretroviral para VIH genera dislipidemia asociada a riesgo cardiovascular y aterosclerosis. Objetivo: Comparar los efectos lipídicos de los antirretrovirales en pacientes con VIH-sida. Métodos: Cohorte retrospectiva. Se comparó el perfil lipídico de los pacientes que recibieron efavirenz (EFV) versus atazanavir (ATV) con una backbone de zidovudina + lamivudina durante 36 meses. Resultados: Se incluyeron 212 pacientes. Desde el inicio hasta los 36 meses, el aumento del HDL del grupo de pacientes en tratamiento con ATV fue mayor en comparación con el que presentaron los pacientes con EFV (8.33 versus 4.26, respectivamente; $p<0.01$ ); se observó una diferencia de triglicéridos entre los grupos, con disminución de $19.06 \mathrm{mg} / \mathrm{dL}$ en los pacientes con ATV y aumento de $40.62 \mathrm{mg} / \mathrm{dL}$ en los que recibieron EFV $(p<0.001)$. $L a$ diferencia de medias en el cambio de colesterol total y $L D L$ entre ambos tratamientos no fue significativa ( $p=0.32$ y $p=0.951$, respectivamente). Conclusiones: Los regímenes con ATV se asociaron a cambios más favorables en los niveles de triglicéridos y $H D L$ que los regímenes con EFV, relación que podría asociarse a reducción del riesgo cardiovascular a largo plazo, la cual requiere estudios adicionales.

PALABRAS CLAVE: Agentes antirretrovirales. Efavirenz. Sulfato de atazanavir. VIH. Perfil lipídico.

\section{Lipid profile levels in HIV-AIDS patients on treatment with efavirenz and atazanavir. Cohort study}

\section{Abstract}

Introduction: Antiretroviral treatment for HIV generates dyslipidemia, which is associated with cardiovascular risk and atherosclerosis. Objective: To compare antiretroviral agents effects on lipids in patients with HIV-AIDS. Methods: Retrospective cohort. The lipid profiles of patients receiving efavirenz (EFV) vs. atazanavir (ATV) with a zidovudine + lamivudine backbone for 36 months were compared. Results: 212 patients were included. From baseline to month 36, HDL increase in the group of patients treated with ATV was higher in comparison with that of patients on EFV (8.33 vs. 4.26; $p<0.01)$; a difference in triglycerides was observed between groups, with a decrease of $19.06 \mathrm{mg} / \mathrm{dL}$ in patients on ATV and an increase of $40.62 \mathrm{mg} /$ $d L$ in those who received EFV $(p<0.001)$. Mean difference in total and $L D L$-cholesterol change between both treatments was not significant ( $p=0.32$ and $p=0.951$, respectively). Conclusions: ATV-containing regimens were associated with more favorable changes in triglyceride and HDL levels than EFV regimens. This benefit could be associated with a reduction in longterm cardiovascular risk; this relationship requires further study.

KEY WORDS: Antiretroviral agents. Efavirenz. Atazanavir sulfate. HIV. Lipid profile.

Correspondencia:

*Maryori M. Hilario-Gómez

E-mail: maryorimhg010514@gmail.com
Gac Med Mex. 2021;157:398-404

Disponible en PubMed

www.gacetamedicademexico.com 0016-3813/@ 2021 Academia Nacional de Medicina de México, A.C. Publicado por Permanyer. Este es un artículo open access bajo la licencia CC BY-NC-ND (http://creativecommons.org/licenses/by-nc-nd/4.0/). 


\section{Introducción}

El VIH/sida es una de las enfermedades más destructivas en la humanidad. ' Los fármacos actualmente usados se relacionan con efectos adversos, incluida la dislipidemia, asociada al desarrollo de aterosclerosis; ${ }^{2}$ además, los antirretrovirales aumentan el estrés oxidativo que promueve enfermedad cardiovascular, con todas las consecuencias posteriores, entre ellas la muerte. ${ }^{3}$

El éxito de los medicamentos antirretrovirales podría quedar oculto por su impacto en el perfil lipídico a largo plazo. No existen conclusiones claras sobre las diferencias en los efectos sobre los lípidos de los diferentes antirretrovirales. ${ }^{4,5}$ Dado que en Perú no hay publicaciones sobre este tema y son pocos los que comparan específicamente efavirenz y atazanavir, en la presente investigación se busca compararlos en asociación con zidovudina y lamivudina (3TC) respecto a los cambios que producen en el perfil de lípidos durante 36 meses.

\section{Métodos}

Se realizó un estudio de cohorte retrospectivo en el que se comparó el perfil lipídico de los pacientes que recibieron efavirenz versus atazanavir con una combinación base de dos inhibidores nucleósidos (backbone) de zidovudina y lamivudina durante 36 meses. Atazanavir fue el tratamiento de elección normado para rescate en los años que se recopiló la información del estudio, recomendado en las guías nacionales de 2005 a 2015. ${ }^{6,7}$ Actualmente se continúa usando en los pacientes que recibieron atazanavir en años anteriores.

Se seleccionó a pacientes que participaron en el programa de prevención y control de ETS/VIH-sida del Hospital "Víctor Lazarte Echegaray", Trujillo, Perú, quienes recibieron tratamiento antirretroviral durante el periodo 2005-2016, mayores de 18 años, de uno y otro sexo, que tuvieran mediciones de control basales (antes del inicio de tratamiento), con al menos seis mediciones de control semestrales en forma continua y que mantuvieron constante y regularmente el esquema de tratamiento asignado, por lo menos durante el periodo de estudio.

Se utilizó un muestreo censal en el que participaron 352 pacientes que recibieron un régimen antirretroviral. La cohorte constó de dos grupos. Los participantes en el primer grupo fueron pacientes mayores de
18 años que comenzaron el tratamiento con efavirenz en combinación con zidovudina y lamivudina y con registros de perfil lipídico durante los tres años de tratamiento. Los participantes en el segundo grupo tuvieron las mismas características, solo que el efavirenz reemplazó al atazanavir. Se excluyeron los pacientes con insuficiencia renal crónica en estadio 3 o más, diabetes mellitus tipos 1 y 2, obesidad y tratamiento con medicamentos reductores de lípidos durante más de seis meses. Durante los años de recolección de información, en el hospital no se contaba con carga viral de VIH ni con recuento de CD4. Los pacientes seleccionados no tuvieron infecciones ni condiciones oportunistas indicadoras del estadio del sida desde su ingreso a la cohorte ni durante el tiempo considerado en el estudio.

El perfil lipídico se midió antes del inicio del tratamiento, así como a los 12, 24 y 36 meses de seguimiento. La hipercolesterolemia, hipertrigliceridemia y disminución del colesterol-HDL se consideraron dislipidemias. ${ }^{8}$

La recolección de datos se realizó a partir de historias clínicas y resultados de laboratorio registrados en el sistema de gestión del hospital.

Se procedió a revisar la base de datos de los pacientes, verificar el tipo de tratamiento que recibieron y formar los grupos que recibirían efavirenz y atazanavir. Posteriormente, se consolidó la información en una hoja de cálculo en SPSS versión 21 para el análisis estadístico correspondiente.

Las variables cualitativas fueron reportadas como frecuencias y porcentajes. Para las variables cuantitativas, al tener una muestra limitada y con distribución no normal según la prueba no paramétrica de Kolmogorov-Smirnov, se calculó la media y la desviación estándar. Se utilizó la prueba t de Student para muestras relacionadas con el fin de determinar el momento en el que se produjo el cambio en cada componente del perfil de lípidos y la diferencia entre la medición basal y la última. Asimismo, la prueba $t$ para muestras independientes sirvió para comparar los cambios producidos en los componentes del perfil de lípidos entre ambos esquemas de tratamiento. Un valor de $p<0.05$ se consideró estadísticamente significativo.

Se aseguró la confidencialidad de la información de cada paciente, para lo cual solo se asignaron códigos a cada entrevista, con previo consentimiento informado. Se dispuso de la autorización del comité de investigación y ética del hospital. 


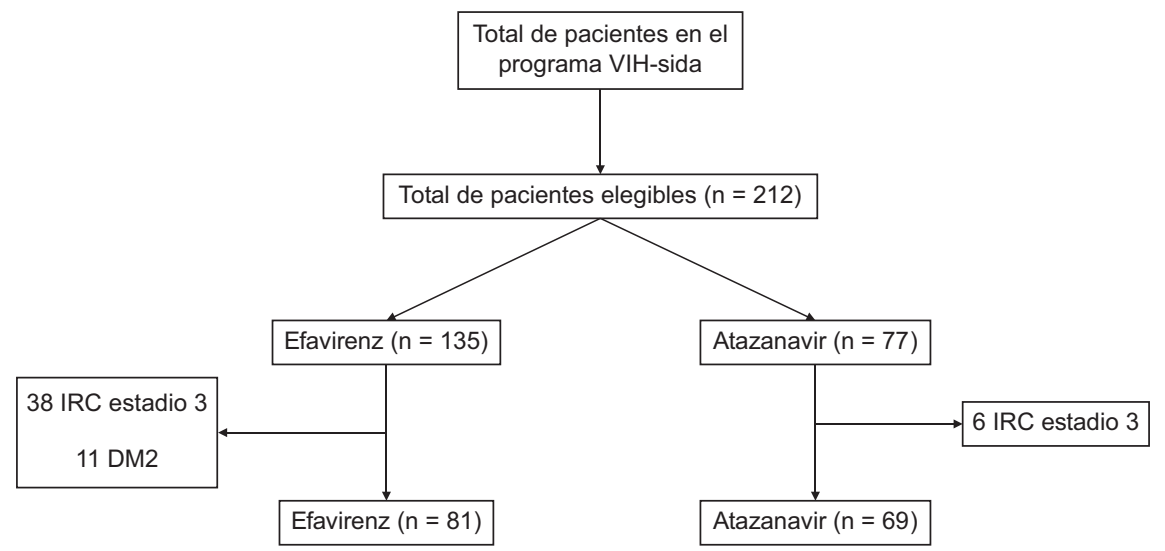

Figura 1. Selección de la población-muestra según los criterios de inclusión y exclusión. $I R C=$ insuficiencia renal crónica, $D M=$ diabetes mellitus.

Tabla 1. Características clínicas y epidemiológicas de los pacientes con VIH-sida

\begin{tabular}{|c|c|c|c|c|c|c|c|c|c|c|c|c|}
\hline \multirow[t]{3}{*}{ Parámetro } & \multicolumn{12}{|c|}{ Perfill lipídico } \\
\hline & \multicolumn{3}{|c|}{ Promedio basal } & \multicolumn{3}{|c|}{ Promedio a los 12 meses } & \multicolumn{3}{|c|}{ Promedio a los 24 meses } & \multicolumn{3}{|c|}{ Promedio a los 36 meses } \\
\hline & $\begin{array}{c}\text { EFV } \\
(n=81)\end{array}$ & $\begin{array}{c}\text { ATV } \\
(n=69)\end{array}$ & p & $\begin{array}{c}\text { EFV } \\
(n=81)\end{array}$ & $\begin{array}{c}\text { ATV } \\
(n=69)\end{array}$ & $\mathrm{p}$ & $\begin{array}{c}\text { EFV } \\
(n=81)\end{array}$ & $\begin{array}{c}\text { ATV } \\
(n=69)\end{array}$ & $\mathrm{p}$ & $\begin{array}{c}\text { EFV } \\
(\mathrm{n}=\mathbf{8 1})\end{array}$ & $\begin{array}{c}\text { ATV } \\
(n=69)\end{array}$ & p \\
\hline $\begin{array}{l}\text { Colesterol } \\
\text { total }^{*}(\mathrm{mg} / \mathrm{dL})\end{array}$ & 195.77 & 180.37 & 0.000 & 193.59 & 182.50 & 0.018 & 200.37 & 189.86 & 0.036 & 208.33 & 189.11 & 0.000 \\
\hline $\mathrm{LDL}^{*}(\mathrm{mg} / \mathrm{dL})$ & 107.60 & 96.17 & 0.046 & 103.97 & 95.98 & 0.109 & 106.86 & 97.75 & 0.074 & 110.37 & 98.73 & 0.023 \\
\hline $\mathrm{HDL}^{*}(\mathrm{mg} / \mathrm{dL})$ & 41.45 & 37.71 & 0.073 & 42.95 & 41.75 & 0.677 & 43.13 & 43.02 & 0.945 & 45.77 & 46.04 & 0.894 \\
\hline $\begin{array}{l}\text { Triglicéridos* } \\
\text { (mg/dL) }\end{array}$ & 163.23 & 200.52 & 0.027 & 168.16 & 187.28 & 0.125 & 180.87 & 180.08 & 0.961 & 186.69 & 173.40 & 0.199 \\
\hline
\end{tabular}

${ }^{*}$ Media obtenida de prueba t de muestras independientes.

$\mathrm{LDL}=$ low-density lipoproteins, $\mathrm{HDL}=$ high density lipoproteins, $\mathrm{EFV}=$ efavirenz, $\mathrm{ATZ}=$ = atazanavir.

Se realizó prueba de t de Student para muestras independientes.

\section{Resultados}

De los 352 pacientes, solo 212 cumplieron con los criterios de inclusión. Hubo 135 pacientes en el grupo tratado con EFV, de los cuales 54 fueron excluidos. Por otro lado, se obtuvieron 77 pacientes del grupo tratado con ATV, de los cuales ocho fueron eliminados (Figura 1).

La edad media global de los pacientes fue de $39.97 \pm 10.92$ años. No se encontraron diferencias significativas en los grupos con EFV y ATV en relación con la edad (39.11 \pm 10.89 versus $40.99 \pm 10.73)$ y el sexo (56\% versus $44 \%$ ), respectivamente (Tabla 1).

La Tabla 2 muestra los promedios de los valores basales de colesterol total, colesterol-LDL, colesterol HDL y triglicéridos de los grupos que recibieron tratamiento con EFV y ATV. Los niveles iniciales de colesterol total y LDL fueron significativamente más altos en los pacientes tratados con EFV en comparación con los tratados con ATV $(p<0.05)$. Los valores de triglicéridos fueron sustancialmente mayores en los pacientes tratados con ATV que en los tratados con EFV $(p<0.05)$.

En cuanto al colesterol total, en los pacientes que recibieron ATV mostró un cambio significativo a los 24 meses $(+9.49 \mathrm{mg} / \mathrm{dL}, \mathrm{p}<0.01)$, mientras que en los pacientes con EFV el aumento fue significativo solo a los 36 meses $(+12.56 \mathrm{mg} / \mathrm{dL}, p<0.01)$. Los valores de colesterol-LDL no mostraron cambios significativos durante el periodo de observación en ninguno de los grupos de tratamiento.

Respecto a los valores de HDL, el grupo tratado con EFV mostró un aumento significativo de $1.68 \mathrm{mg} / \mathrm{dL}$ a los 24 meses $(\mathrm{p}<0.01)$ y de $+4.04 \mathrm{mg} / \mathrm{dL}$ 
Tabla 2. Promedio de perfil lipídico basal, a los 36 meses y cambio a lo largo del periodo de observación

\begin{tabular}{|c|c|c|c|c|}
\hline Variables & $\begin{array}{c}\text { Población } \\
\text { total } \\
(n=150)\end{array}$ & $\begin{array}{c}\text { EFV } \\
(n=81) \\
(54 \%)\end{array}$ & $\begin{array}{c}\text { ATV } \\
(n=69) \\
(46 \%)\end{array}$ & $p$ \\
\hline > 40 años & 73 & $37(50)$ & $36(50)$ & 0.428 \\
\hline$<40$ años & 77 & $44(57.3)$ & $33(42.7)$ & \\
\hline $\begin{array}{l}\text { Sexo } \\
\text { masculino }\end{array}$ & 116 & $65(56.0)$ & $51(44.0)$ & 0.35 \\
\hline $\begin{array}{l}\text { Sexo } \\
\text { femenino }\end{array}$ & 34 & $16(47.0)$ & $18(53.0)$ & \\
\hline Origen latino & 150 & $81(54)$ & $69(46)$ & \\
\hline $\begin{array}{l}\text { Edad en } \\
\text { años } \\
\text { (media } \pm D E \text { ) }\end{array}$ & $\begin{array}{c}39.97 \\
\pm 10.92\end{array}$ & $\begin{array}{c}39.11 \\
\pm 10.89\end{array}$ & $\begin{array}{c}40.99 \\
\pm 10.73\end{array}$ & 0.292 \\
\hline
\end{tabular}

a los 12 meses $(p<0.01)$ en ATV. Asimismo, en relación con los valores de triglicéridos, los pacientes tratados con EFV presentaron un aumento significativo a los 12 meses $(+4.93 \mathrm{mg} / \mathrm{dL}, p<0.01)$. En contraste, los pacientes en el grupo con ATV mostraron una disminución significativa a los 24 meses (-11.94 mg/dL, $p<0.05)$ (Figura 2).

Finalmente, se realizó una comparación de los cambios en el perfil lipídico medio a los 36 meses en ambos grupos. El aumento en la media de los valores iniciales de HDL a los 36 meses fue mayor en el grupo tratado con ATV en comparación con los pacientes tratados con EFV $(p<0.01)$. No se encontraron valores significativos en la diferencia de medias a los 36 meses en colesterol total y LDL en los grupos tratados con EFV y ATV. Respecto a la media de triglicéridos a los 36 meses en el grupo EFV, se observó aumento de la media en 4.62 y reducción de la media en 19.06 en el grupo con ATV $(p<0.001)$ (Figura 3$)$.

\section{Discusión}

Nuestros hallazgos sugieren que los regímenes antirretrovirales que contienen ATV no producen alteraciones significativas en el perfil lipídico en comparación con EFV.

En los pacientes con VIH, el uso de algunos antirretrovirales, especialmente los inhibidores de proteasa, ha implicado el desarrollo de disfunciones endoteliales, mitocondriales y celulares, así como el aumento del factor de necrosis tumoral- $\alpha$ y las interleucinas 1 y 6 , que favorecen aún más el estado lipogénico. ${ }^{9}$ La dislipidemia es una complicación a largo plazo relacionada con aumento significativo de la tasa de mortalidad asociada a eventos cardiovasculares.

La incidencia de dislipidemia de nuestra serie fue similar a la encontrada en otro estudio efectuado en Perú, donde Rondan et al. encontraron una prevalencia de $74.7 \% .{ }^{10}$ Asimismo, en un análisis que incluyó a más de 4000 pacientes de siete países latinoamericanos, $80.2 \%$ de la dislipidemia general y $67.9 \%$ de la dislipidemia se encontraron en pacientes peruanos. ${ }^{10,11}$

Los pacientes que recibieron ATV tuvieron disminución favorable de los triglicéridos después de 12 meses de tratamiento, evidente a los 36 meses de tratamiento. El colesterol-HDL tuvo un aumento que comenzó a los 12 meses de tratamiento y fue aumentando hacia el periodo final de observación. Estos resultados son comparables con el estudio realizado por Carey et al., quienes utilizaron ATV con un backbone de AZT + 3TC, lo cual produjo cambios favorables en las HDL. Los pacientes en el estudio mencionado utilizaron ATV solo o con ritonavir; se demostró que el último no influye en los niveles de LDL, HDL o triglicéridos. ${ }^{12}$

Nuestro análisis indicó que después de 36 meses de tratamiento, los pacientes con ATV tuvieron cambios más favorables en el perfil lipídico, lo que traduce un beneficio esencial respecto a los triglicéridos y el colesterol-HDL. Ambos grupos mostraron aumento en el colesterol total, más altos en el grupo con EFV (+12.56 mg/dL versus $8.33 \mathrm{mg} / \mathrm{dL})$, pero sin diferencia estadísticamente significativa. Por otro lado, no se encontraron cambios significativos en las LDL en ninguno de los grupos.

Respecto a los triglicéridos, nuestros resultados coinciden con los de Squires et al., quienes en un estudio aleatorizado doble ciego con 810 pacientes encontraron una reducción de $9 \%$ en los triglicéridos a favor de ATV con el mismo backbone que empleamos; ${ }^{13}$ además, Daar et al., en un estudio aleatorizado de 685 pacientes, compararon EFV y ATV en el que utilizaron backbone de tenofovir emtricitabina y abacavir-lamivudina; no encontraron diferencias significativas en los triglicéridos. ${ }^{14}$

Identificamos que tanto EFV como ATV producen este importante cambio beneficioso en el HDL, con alguna ventaja para el grupo que recibió el segundo.

Se conoce la relación con la terapia antirretroviral altamente activa con un patrón de colesterol-HDL 

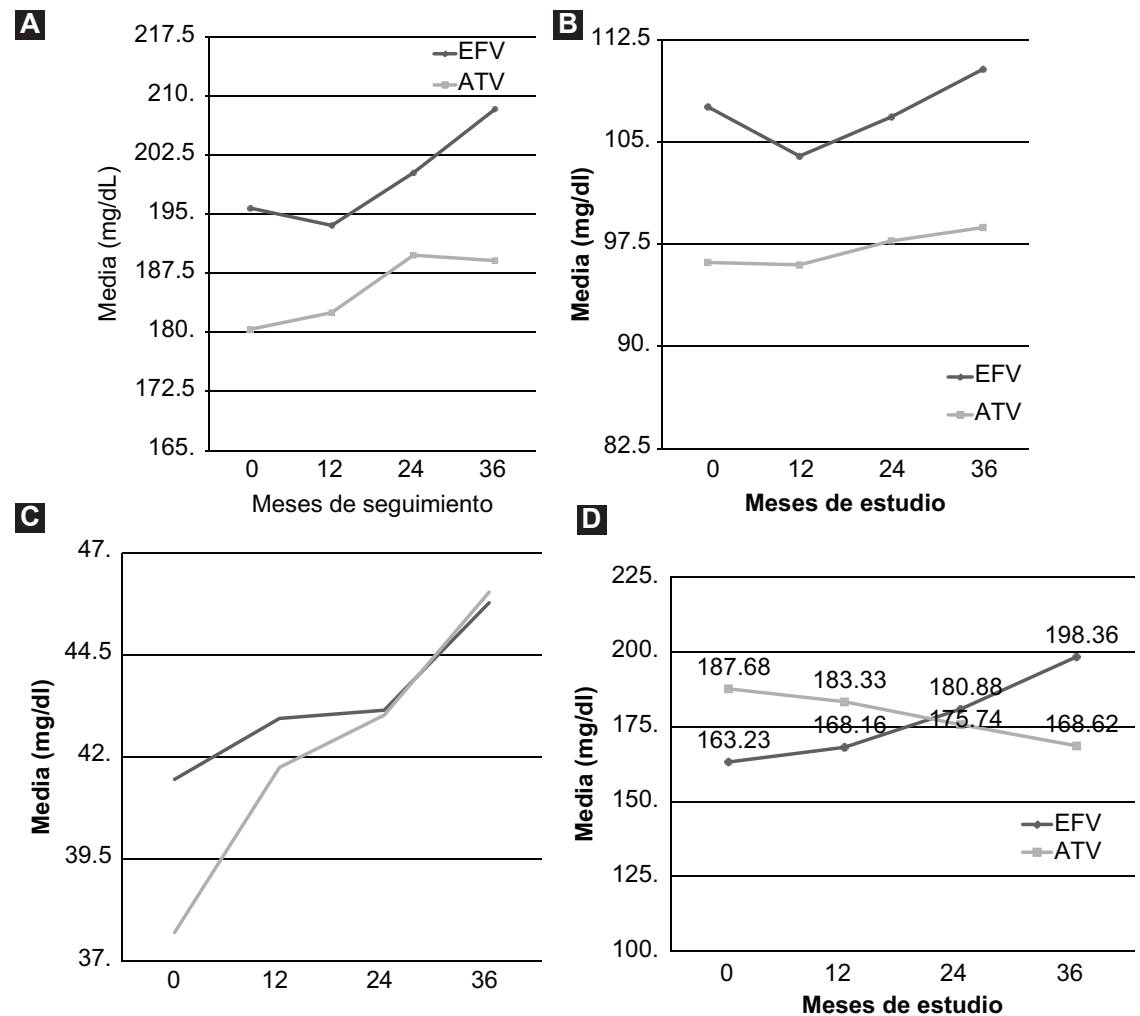

Figura 2. A) Colesterol total durante todo el periodo de estudio con efavirenz (EFV) versus atazanavir (ATV). B) LDL a lo largo del periodo de estudio. C) HDL. D) Triglicéridos.

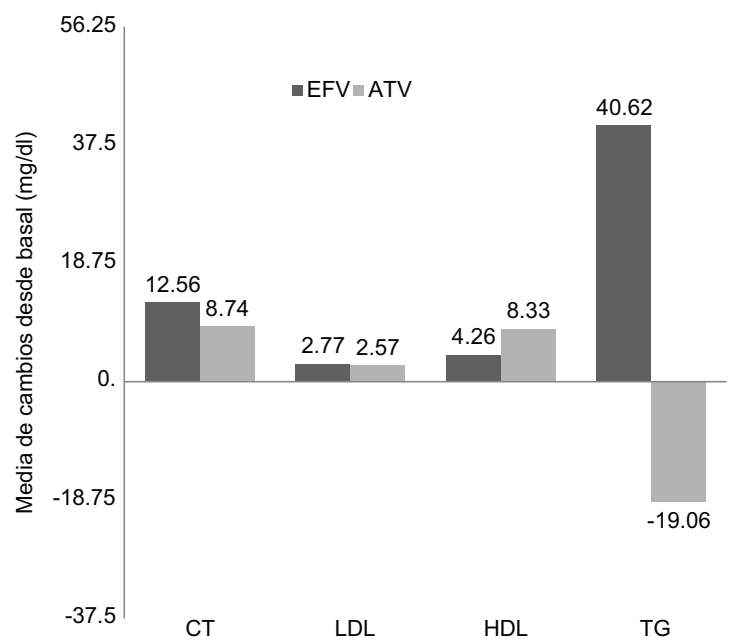

Figura 3. Triglicéridos durante el periodo de estudio con efavirenz (EFV) versus atazanavir. Se utilizó prueba t para muestras relacionadas.

bajo y triglicéridos elevados, como lo indican Estrada y Portilla, ${ }^{15}$ al cual se denomina "dislipidemia aterogénica"; entre estos cambios, los bajos niveles de HDL (<35 mg/dL) están directamente relacionados con la mortalidad por enfermedad cardiovascular ( $\mathrm{RM}=22.92, \mathrm{p}=0.03$ ) en pacientes con $\mathrm{VIH} .{ }^{16}$ Se ha registrado reducción de hasta $60 \%$ en el riesgo cardiovascular en pacientes que reciben ATV. ${ }^{17}$ La relación entre el uso de ATV y el efecto beneficioso sobre el grosor de la íntima y los medios de comunicación de la carótida es otro dato a favor de su buen perfil

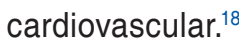

Los resultados encontrados por Estrada et al., respecto a que el estado proinflamatorio y oxidativo (medido por las concentraciones séricas de fosfolipasa A2, mieloperoxidasa), es menor en pacientes con esquemas basados en ATV en comparación con $\mathrm{EFV},{ }^{19}$ también podrían explicar el beneficio clínico en el perfil lipídico observado con ATV.

La principal fortaleza de este estudio fue el seguimiento de los pacientes durante 36 meses, el cual tenía como objetivo primario evaluar los cambios en el perfil lipídico, a diferencia de la mayoría de las investigaciones anteriores que abordaron el perfil lipídico como resultado secundario. Dado que la etiopatogenia de la dislipidemia en el VIH es multifactorial y también está asociada a factores étnicos, 
socioeconómicos y culturales, este estudio es muy importante porque es el primero de este tipo que se realiza en nuestro país. ${ }^{20}$

Una limitación del presente análisis es que el fenómeno aterogénico puede expresarse en diferentes momentos dependiendo de la genética de los pacientes. Si bien otra limitante importante fue no tener la carga viral y el recuento de CD4, Kelesidis et al. observaron que el uso de inhibidores de proteasa se asoció con dislipidemia, independientemente de la carga viral, el recuento de linfocitos CD4, el sexo e, incluso, otros factores de riesgo cardiovascular tales como fumar; ${ }^{21}$ de ahí que el desenlace clínico quedaría como un patrón surrogado con esta evidencia. Por ello, en el presente estudio la interpretación de los resultados del seguimiento de los parámetros laboratoriales (perfil lipídico) debía efectuarse exclusivamente en el contexto de exposición a dos clases diferentes de fármacos antirretrovirales, ya que el eje principal del régimen era el mismo en ambos grupos (zidovudina/lamivudina). En las historias clínicas de los pacientes incluidos en este estudio no se encontró alusión al uso de fármacos reductores de lípidos y no fue posible corroborar con una encuesta posterior si los pacientes recibieron estos fármacos.

La gravedad de la dislipidemia varía según el esquema utilizado. Por esta razón, el uso de los inhibidores de proteasa ya está incluido como un factor en algunas escalas de riesgo cardiovascular. ${ }^{22}$ En el presente estudio, uno de los comparadores se utilizó como inhibidor de la proteasa, atazanavir, pero los casos seleccionados para el análisis fueron aquellos en los que no se utilizó junto con ritonavir. A diferencia de lo anterior, en el análisis de Rondón se registró fuerte asociación entre la dislipidemia y el uso de inhibidores de proteasa, pero los inhibidores de proteasa utilizados fueron lopinavir/ritonavir, atazanavir y ritonavir. Por lo tanto, el panel de expertos del Departamento de Salud y Servicios Sociales de los Estados Unidos (DHHS) ${ }^{23}$ señala que todos los inhibidores de proteasa potenciados con ritonavir aumentan los niveles de colesterol HDL y LDL, así como los triglicéridos, lo cual explica la diferencia con otras series que no compararon los niveles de colesterol y triglicéridos con ATV no aumentado con ritonavir, como se hizo en el presente estudio.

\section{Conclusiones}

Esta investigación ha mostrado que ATV sin refuerzo produjo cambios lipídicos más favorables que EFV en la población de estudio, lo que podría estar asociado a una disminución a largo plazo en el riesgo cardiovascular.

\section{Financiamiento}

La presente investigación no recibió ninguna beca específica de agencias de los sectores públicos, comercial o sin ánimo de lucro.

\section{Conflicto de intereses}

Los autores declaran no tener conflicto de intereses alguno.

\section{Responsabilidades éticas}

Protección de personas y animales. Los autores declaran que para esta investigación no se realizaron experimentos en seres humanos ni en animales.

Confidencialidad de los datos. Los autores declaran que siguieron los protocolos de su centro de trabajo sobre la publicación de datos de pacientes.

Derecho a la privacidad y consentimiento informado. Los autores obtuvieron el consentimiento informado de los pacientes o sujetos referidos en el artículo. Este documento obra en poder del autor de correspondencia.

\section{Bibliografía}

1. Sárcamo CC, Blitchtein-Winicki D, Valverde RA, Best RJ, Suárez-Ognio L, Campos GJ, et al. Estudio basal de prevalencia de sífilis y VIH y comportamientos asociados en población privada de libertad, Perú 1999. Rev Peru Med Exp Salud Publica. 2003:20:9-14.

2. Rodríguez-Vidigal FF, Muñoz-Sanz A. Alteraciones del metabolismo de los lípidos en los pacientes infectados por el virus de la inmunodeficiencia humana. Una hipótesis acerca de su patogenia. Med Clin. 2000;115:145-150.

3. Roca B. Trastornos metabólicos relacionados con el VIH y el tratamiento antirretroviral. An Med Interna (Madrid). 2003;20:37-45.

4. Guevara FO, Cañón B, Liévano MC, Lombo B, Rendón I, Blanco F. Prevalence of metabolic syndrome in patients infected with HIV. Acta Med Colombiana. 2008;33:7.

5. Ministerio de Salud. Norma técnica de salud de atención integral del adulto con infección por el virus. Peru: Ministerio de Salud; 2018.

6. Geldres-Molina F. Niveles de perfil lipídico en pacientes con VIH-SIDA en tratamiento con efavirenz y atazanavir. EsSalud. La Libertad. 20052016. Perú: Universidad Privada Antenor Orrego; 2017.

7. Ministerio de Salud. Norma técnica de salud de atención integral del adulto con infección por el virus de la inmunodeficiencia humana. Perú: Ministerio de Salud; 2015.

8. Pallarés-Carratalá V, Pascual-Fuster V, Godoy-Rocatí D. Dislipidemia y riesgo vascular. Una revisión basada en nuevas evidencias. Semergen. 2015:41:435-445.

9. Pacífico J, Gutiérrez C. Información sobre la medicación y adherencia al tratamiento antirretroviral de gran actividad en pacientes con $\mathrm{VIH} /$ sida de un hospital de Lima, Perú. Rev Peru Med Exp. 2015;32:66-72.

10. Rondán PL, Flores-Flores O, Doria NA, Valencia-Mesías G, Chávez-Pérez V Soria J. Elevada frecuencia de dislipidemia en pacientes infectados por VIH en un hospital público peruano. Rev Peru Med Exp. 2017:34:239-244.

11. Cahn P, Leite O, Rosales A, Cabello R, Álvarez CA, Seas C, et al. Metabolic profile and cardiovascular risk factors among Latin American HIV-infected patients receiving HAART. Braz J Infect Dis. 2010;14:158-166. 
12. Carey D, Amin J, Boyd M, Petoumenos K, Emery S. Lipid profiles in HIV-infected adults receiving atazanavir and atazanavir/ritonavir: systematic review and meta-analysis of randomized controlled trials. J Antimicrob Chemother. 2010;65:1878-1888.

13. Squires KE, Young B, Dejesus E, Bellos N, Murphy D, Zhao HH, et al. Similar efficacy and tolerability of atazanavir compared with atazanavir/ ritonavir, each with abacavir/lamivudine after initial suppression with abacavir/lamivudine plus ritonavir-boosted atazanavir in HIV-infected patients. AIDS. 2010;24:2019-2027.

14. Daar ES, Tierney C, Fischl MA, Sax PE, Mollan K, Budhathoki C, et al. Atazanavir plus ritonavir or efavirenz as part of a 3-drug regimen for initial treatment of HIV-1. Ann Intern Med. 2011;154:445-456.

15. Estrada V, Portilla J. Dyslipidemia related to antiretroviral therapy. AIDS Rev. 2011;13:49-56

16. Jain N, Tripathi AK, Vaish AK, Verma SP, Himanshu D, Gutch M. Can metabolic factors be used prognostically for short-term mortality in HIV-infected patients? Ann Med Health Sci Res. 2012;2:124-128.

17. Díaz CM, Segura ER, Luz PM, Clark JL, Ribeiro SR, De Boni R, et al Traditional and HIV-specific risk factors for cardiovascular morbidity and mortality among HIV-infected adults in Brazil: a retrospective cohort study. BMC Infect Dis. 2016;16:376.
18. Chow D, Shikuma C, Ritchings C, Guo M, Rosenblatt L. Atazanavir and cardiovascular risk among human immunodeficiency virus-infected patients: a systematic review. Infect Dis Ther. 2016;5:473-489.

19. Estrada V, Monge S, Gómez-Garre D, Sobrino P, Berenguer J, Bernardino $\mathrm{Jl}$, et al. Comparison of oxidative stress markers in HIV-infected patients on efavirenz or atazanavir/ritonavir-based therapy. J Int AIDS Soc. 2014;17:19544.

20. Pozniak AL, Gallant JE, DeJesus E, Arribas JR, Gazzard B, Campo RE, et al. Tenofovir disoproxil fumarate, emtricitabine, and efavirenz versus fixed-dose zidovudine/lamivudine and efavirenz in antiretroviral-naive patients: virologic, immunologic, and morphologic changes--a 96-week analysis. J Acquir Immune Defic Syndr. 2006;43:535-540.

21. Kelesidis T, Currier JS. Dyslipidemia and cardiovascular risk in human immunodeficiency virus infection. Endocrinol Metab Clin North Am. 2014:43:665-684.

22. Friis-Møller N, Ryom L, Smith C, Weber R, Reiss P, Dabis F, et al. An updated prediction model of the global risk of cardiovascular disease in HIV-positive persons: The Data-collection on Adverse Effects of Anti-HIV Drugs (D: A:D) study. Eur J Prev Cardiol. 2016;23:214-223.

23. NIH. What's New Adult and Adolescent Opportunistic Infection. AIDSinfo; 2019 